\title{
Using A Functional Play Intervention to Increase Joint Attention of School-aged, Non-Verbal Children with Autism Spectrum Disorder (ASD)
}

\author{
Taylor Basso a, Marjorie H. Charlop ${ }^{b}$, Caitlyn B. Gumaerc,
}

$\begin{array}{ll}\text { Received } & : \text { 18 June } 2020 \\ \text { Revised } & : \text { 23 October } 2020 \\ \text { Accepted } & : 5 \text { January } 2021 \\ \text { DOI } & : \text { 10.26822/iejee.2021.193 }\end{array}$

a Taylor Basso,

Claremont McKenna College, California, USA. E-mail: tbasso14@students.claremontmckenna.edu

${ }^{b}$ Marjorie H. Charlop, Claremont McKenna College, California, USA.

E-mail: Marjorie.Charlop@ClaremontMcKenna.edu ORCID: https://orcid.org/0000-0001-7208-0116

-Correspondance Details: Caitlyn B. Gumaer, Department of Psychological Sciences, Claremont McKenna College., 850 Columbia Avenue, Claremont, USA. E-mail: Caitlyn.Gumaer@ClaremontMcKenna.edu ORCID: https://orcid.org/0000-0002-9682-0729

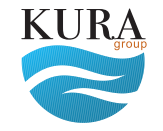

Copyright $@$

www.iejee.com

ISSN: $1307-9298$

(C) 2021 Published by KURA Education \& Publishing. This is an open access article under the CC BYNC- ND license. (https://creativecommons.org/ licenses/by/4.0/)

\begin{abstract}
The vast majority of joint attention interventions for children with autism spectrum disorder (ASD) are geared toward toddlers and preschoolers as it is an early developmental skill. However, many of the youngsters do not acquire joint attention despite these early interventions and subsequently do not exhibit joint attention later in life. In the current study, we used a multiple baseline design across three school-aged, non-verbal children with ASD, to assess the efficacy of a functional play intervention to increase joint attention. During baseline, all three children demonstrated minimal joint attention and appropriate play. Following baseline, the functional play intervention was presented, which consisted of teaching functional play through modeling. Post-intervention probes demonstrated gains in both joint attention and functional play. Furthermore, these behaviors generalized across person, setting, and stimuli. The results of this study provide evidence that functional play interventions may be a promising approach to teach and promote joint attention for older, school-aged children with ASD.
\end{abstract}

\section{Keywords:}

Joint Attention, Play, Autism Spectrum Disorder

\section{Introduction}

hildren with autism spectrum disorder (ASD) typically have underdeveloped joint attention skills. Joint attention is the use of verbal (i.e., commenting, question asking) and nonverbal (i.e., eye-gazing, gesturing) forms of communication to coordinate between a socialcommunicative partner and an object or event of interest, with the intent of sharing that experience (MacDonald et al., 2006; Miendl \& Cannella-Malone, 2011; Mundy, Sigman, \& Kasari, 1994; White et al., 2011). Recognized as one of the earliest forms of communication, joint attention is considered a foundational skill associated with the development of language, play, imitation, and social behavior in both typically developing children (Baron-Cohen, 1991) and children with ASD (Mundy et al., 2007). Interventions teaching joint attention to children with ASD have primarily been conducted with toddlers and preschool-aged 


\section{iejee $\approx$}

children with ASD, not school-aged children (Miendl \& Cannella-Malone, 2011; Murza et al., 2016; White et al., 2011). Unfortunately, joint attention often stops being a target of intervention in older, school-aged children with ASD. The literature has shown that young children with the least amount of language (e.g., nonverbal or minimally verbal) have benefitted the most from joint attention interventions (Kasari et al., 2008; Kasari et al., 2010), thus targeting joint attention in the treatment of older children with ASD needs to be addressed.

In the past few decades, interventions that specifically target joint attention have been developed for toddlers and preschool children with ASD (Miendl \& CannellaMalone, 2011; Murza et al., 2016; White et al., 2011). Three review articles have assessed a number of these interventions (Miendl \& Cannella-Malone, 2011; Murza et al., 2016; White et al., 2011). These interventions vary in terms of the implementation of the procedures (e.g., parent-training, physical prompting) and the degree to which social versus non-social (i.e., tangibles) consequences were used to increase joint attention behaviors (Miendl \& Cannella-Malone, 2011). The review suggested that the majority of interventions were effective in increasing joint attention skills in very young children with ASD.

Researchers have proposed that for these children with ASD, joint attention needs to be the direct target within interventions to robustly affect change (Kasari et al., 2006; Whalen \& Schreibman, 2003; White et al., 2011). A variety of treatment packages have been used including least-to-most prompting, verbal prompting, with edible and social reinforcers (e.g., praise; Taylor \& Hoch, 2008). Typically, joint attention interventions range from 70 to 90 sessions, including baseline and generalization probe measurements. Many of these interventions also reported increases in ancillary measures of other social behaviors such as spontaneous speech, expressive language, socialcommunicative behaviors, and play (Jones et al., 2006; Kasari et al., 2008; Miendl \& Cannella-Malone, 2011; Whalen \& Schreibman, 2003; White et al., 2011). White and colleagues (2011) reported that in 18 of the articles they reviewed, a play context was used for teaching joint attention, suggesting that joint attention and play go together. However, for children with ASD the use of nonsocial consequences, edible or other tangible reinforcers are often required (Miendl \& Cannella-Malone, 2011; White et al., 2011). In Miendl and Cannella-Malone's (2011) systematic review, a large number of studies suggested the need for tangible reinforcers and/or access to preferred activities or items as consequences for joint attention. In almost all of the successful interventions targeting joint attention, direct reinforcement was used (White et al., 2011).

Taken together, the results of prior studies suggest that play should somehow be related to the teaching of joint attention (White, et al., 2011) and that direct tangible reinforcement should be an element of the intervention process (Miendl \& Cannella-Malone, 2011). The present study incorporated these two findings into the current treatment. First, rather than merely using the context of play, functional play was used as the means to teach joint attention. It was thought that this would add a motivating element to the intervention (Whalen \& Schreibman, 2003). Second, direct reinforcement in terms of access to the functional play item was provided. Importantly, several expansions to the literature were made in the present research including: (1) the participation of school-aged, non-verbal children with ASD, (2) the participation of various ethnicities, (3) assessment of generalization of training across person, setting, and stimuli, and (4) inclusion of measures of both functional play and joint attention.

\section{Method}

\section{Participants}

Participants included three boys attending weekly behavioral therapy session at an after-school treatment center for children with ASD. All three children received an independent diagnosis of ASD according to the Diagnostic and Statistical Manual of Mental Disorders-5th edition (DSM-5; American Psychiatric Association, 2013), attended elementary school or participated in a specialized education program, and demonstrated limited amounts of joint attention and appropriate play skills. Additional characteristics of the participants were assessed using the Childhood Autism Rating Scale-2 (CARS-2; Schopler, Van Bourgondien, Wellman, \& Love, 2010), and the Vineland Adaptive Behavior Scales-Second Edition (Vineland-II; Sparrow et al., 2005). A summary of participants and their characteristics is presented in Table 1.

Kevin is a South Asian American boy, who was 6 years and 8 months old at the start of baseline. Kevin's adaptive functioning was in the low range with low scores for receptive and expressive language skills, based on parent report (Vineland-Il; Sparrow et al., 2005). Kevin demonstrated a severe delay in the development of play skills, often engaging with toys inappropriately (i.e., stereotypy) or not at all. Additionally, Kevin demonstrated limited joint attention behaviors, often only making eye contact when prompted.

Jordan is a Latinx boy, who was 6 years and 9 months at the beginning of baseline. Based on parent report (Vineland-Il; Sparrow et al., 2005), Jordan's adaptive functioning was in the low range, with low scores in receptive and expressive language skills. Jordan 
Table 1

Partcipant Characterictics

\begin{tabular}{lllll}
\hline Participant & Age & Sex & Ethnicity & VABS-3 Adaptive \\
Level & $\begin{array}{l}\text { CARS-2 ASD Classi- } \\
\text { fication }\end{array}$ \\
\hline Kevin & $6: 8$ & Male & South Asian American & Low \\
\hline Jordan & $6: 9$ & Male & Latinx & Low \\
\hline Brandon & $7: 7$ & Male & Asian American & Moderately Low \\
\hline
\end{tabular}

demonstrated limited receptive and expressive language: he exhibited one-word, spontaneous requests for food and snack items, used full phrases to request only when prompted, and frequently exhibited echolalia rather than functional speech. Jordan lacked motivation for toys and engaged in destructive play or inappropriate mouthing of toys; he often engaged in repetitive and stereotyped behaviors with items used in the study. Jordan had limited joint attention behaviors, and would not make eye contact even when prompted.

At the start of baseline, Brandon, an Asian American boy, was 7 years and 7 months. Brandon's adaptive functioning was in the moderately low range with low scores for receptive and expressive language skills, based on parent report (Vineland-Il; Sparrow et al., 2005). Brandon had some delay in the development of play skills and would not engage in play appropriately. Brandon had limited joint attention behaviors, often not engaging in joint attention and averting eye contact with others.

\section{Materials}

A group of predetermined toys selected based on a preference assessment during a free operant observation procedure (Roane, Vollmer, Ringdahl \& Marcus, 1998), were chosen and used throughout the study. The 20 preselected toys were only used for the study, and were not available outside of the study to prevent bias and ensure that each child was equally exposed to the materials during the study.

\section{Setting}

Baseline sessions, functional toy play sessions, trialbased play probe sessions, and follow-up probes were conducted in a therapy room $(1.5 \mathrm{~m}$ by $3 \mathrm{~m}$ ) located at the after-school program. Generalization probes were conducted by an unfamiliar person in an unfamiliar room ( $1.5 \mathrm{~m}$ by $3 \mathrm{~m}$ ) located near the afterschool program. Both rooms contained a child-sized table and two child-sized chairs, with the child and therapist facing one another and a gray lapboard on the therapist's lap, which was used to present the toys and model appropriate play to the child.

\section{Design}

A single subject multiple baseline design across parti- cipants was used to assess the effects of the functional toy play intervention. Multiple baseline designs are established experimental techniques commonly used in research with children with ASD (Nock, 2002).

The children first completed baseline sessions, which were trial-based play probes sessions consisting of 8 opportunities to engage in joint attention with the therapist and to display functional play skills. After baseline, the trial-based functional toy play intervention and trial-based play probes were introduced in order to facilitate the acquisition of joint attention and functional play skills, thus the intervention included two recorded sessions. Following each functional toy play intervention session, trialbased play probes (similar to baseline sessions) were conducted. Criterion was met when the child engaged in 7 correct joint attention responses out of 8 opportunities across two consecutive trial-based play probe sessions.

\section{Procedure}

\section{Baseline}

Each child's frequency of joint attention and functional toy play occurrences was assessed during an approximately five-minute trial-based recorded play probe sessions. Each play probe consisted of eight opportunities for the child to engage in joint attention and demonstrate functional play skills. The experimenter introduced eight different toys randomly, one at a time from the box of twenty possible highly preferred toys. The experimenter showed the child the toy for approximately 2 seconds, waiting for any joint attention to be displayed. The experimenter then gave the toy to the child for $3-5$ seconds. During this time, the child could interact with the toy anyway he wanted, but was reinforced for functional play with social praise (e.g., "that is good playing"). If at any point during each of these opportunities (trials), the child engaged in joint attention behaviors with the experimenter, the behavior was reinforced with an edible and praise (e.g., "That was good looking, Kevin. You looked at me, at the toy, and back at me. Good job!"). If the child did not engage in joint attention behavior, the experimenter would move on to the next trial by removing and putting away the toy, then introducing a new toy. During these sessions the child was given the opportunity to engage in both functional play and joint attention. 


\section{iejee}

\section{Generalization probes}

To assess generalization of functional play and joint attention, probes were conducted during baseline and following the completion of treatment. Similar to baseline sessions, trial-based play probes were conducted in a play room not associated with the study with an unfamiliar adult and novel toys.

\section{Functional toy play intervention}

Following baseline, the functional toy play intervention was introduced, consisting of the functional toy play intervention sessions and subsequent play probes. These sessions were structured to serve as a close model of typical dyad playing, using phrases such as "Your Turn" and "My Turn" to establish these sessions as interactive play sessions, rather than as an individual play session.

During the intervention sessions, when introducing each toy, the experimenter held the toy at eye level and then put it on the lapboard, allowing the child an opportunity to engage in joint attention behaviors. Then the experimenter would model functional play with the toy three times. The child was then given the opportunity to functionally play with the toy, being prompted by the experimenter with phrases like "Do this" or "Can you make the dinosaur stomp" and "Your turn." If at any time during the trial, the child engaged in joint attention with the experimenter, joint attention was immediately reinforced with an edible, verbal praise, and immediate access to the toy (i.e., access to the toy for an additional 30 seconds). If the child did not engage in joint attention behaviors, such reinforcement did not occur, and the experimenter moved on to the next trial. To conclude a trial, the experimenter said, "My turn" and removed the toy from the lapboard and commenced with the next trial. To summarize, the intervention consisted of: 1) presentation of the toy at eye level, 2) modeling of the functional use of the toy three times consecutively, 3) handing over the toy to the child and allowing the opportunity for functional toy play and joint attention on behalf of the child, and 4) and providing the consequences for joint attention when and if it occurred. Praise for correct functional play was provided.

\section{Play probes}

Each five-minute functional toy play intervention session was immediately followed by a trial-based play probe session, mimicking baseline procedures. Similar to baseline, the child was not prompted to engage in play behaviors and joint attention but was given the opportunity to engage and immediately reinforced with an edible, verbal praise and access to the toy. Mastery criterion was set to 7 correct joint attention responses out of 8 opportunities, across two consecutive trial-based play probe sessions. Play probes were presented to assess if joint attention occurred in a non-training setting.

\section{Dependent Measures}

This study included two measures: joint attention and functional play.

\section{Joint attention}

For the present study, joint attention was operationally defined as a response in which the child exhibits eye contact with the experimenter, gazes at the toy, and regains eye contact with the experimenter within 10 seconds. Also, the child looking at the toy, then looking at the experimenter and then shifting his gaze back to the toy is included in the operational definition.

\section{Functional play}

In this study, functional play was operationally defined as the child either a) appropriately imitating the functional toy play behavior modeled by the experimenter, or (e.g. bouncing the ball), or b) using the toys to perform actions different from those modeled, but still considered a functional and appropriate use for the toy (e.g. rolling the ball).

\section{Scoring/Reliability}

During each phase of the study, an observer video recorded each session. Following data collection, two reliable raters conducted inter-observer agreement by rating $33 \%$ of videotaped sessions during baseline, generalization probes, and appropriate play intervention for each child. The two raters reviewed the operational scoring definitions and followed the same scoring procedure. Inter-observer agreement included calculating the number of agreements (i.e., occurrence and nonoccurrence) divided by the sum of observations (i.e., agreements plus disagreements) multiplied by 100 . Inter-observer agreement was averaged at $97 \%$ for joint attention engagements and $87 \%$ for appropriate play behaviors across all the children across all data collection phases.

\section{Procedural Reliability}

Procedural reliability was assessed to ensure the protocol was implemented without potential bias and to ensure procedural integrity. For each participant, two observers independently rated at least 33\% of videotaped sessions (i.e., baseline, treatment, play probes, generalization probes). Sessions were assessed to determine whether the intended intervention was 
implemented correctly. Sessions were coded using a checklist (made available upon request) designed to measure correct and incorrect application of instructions, opportunity presentation, consistent opportunity length, and absence of additional prompting. Procedural integrity was calculated by dividing the number of correctly implemented steps by the total number of steps and multiplying by 100 . Treatment fidelity was $100 \%$ across all phases and all children.

\section{Results}

All three children with ASD met learning criterion by demonstrating gains in joint attention engagements during intervention of 7 out of 8 opportunities (trials). All children demonstrated joint attention mastery during play probes.The children also demonstrated high frequencies of functional play both during intervention and within post-intervention play probes as compared to baseline. For all three of the children, these gains generalized across person and setting in post-treatment generalization probes. Figure 1 depicts the children's joint attention engagements, and Figure 2 depicts the children's functional toy play behaviors.

\section{Joint Attention}

\section{Kevin}

During baseline, Kevin exhibited very little joint attention (see panel 1, Figure 1). The introduction of the functional play intervention, however, steadily increased Kevin's joint attention across the 8 opportunities during intervention and post play probe sessions. In the intervention phase, Kevin demonstrated a significant increase in joint attention in both the functional play intervention sessions and more importantly in subsequent play probes. Kevin met learning criterion (e.g., joint attention in 7 out of 8 opportunities across 2 consecutive sessions) after 7 functional play intervention sessions. In Kevin's last two intervention sessions, joint attention occurred for 7 out of 8 opportunities. Treatment gains generalized to an unfamiliar setting with an unfamiliar person and untrained stimuli.

\section{Jordan}

Jordan's joint attention (see panel 2, Figure 1), remained consistently low during baseline; however, it immediately increased upon the introduction of the

Figure 1

Joint Attention

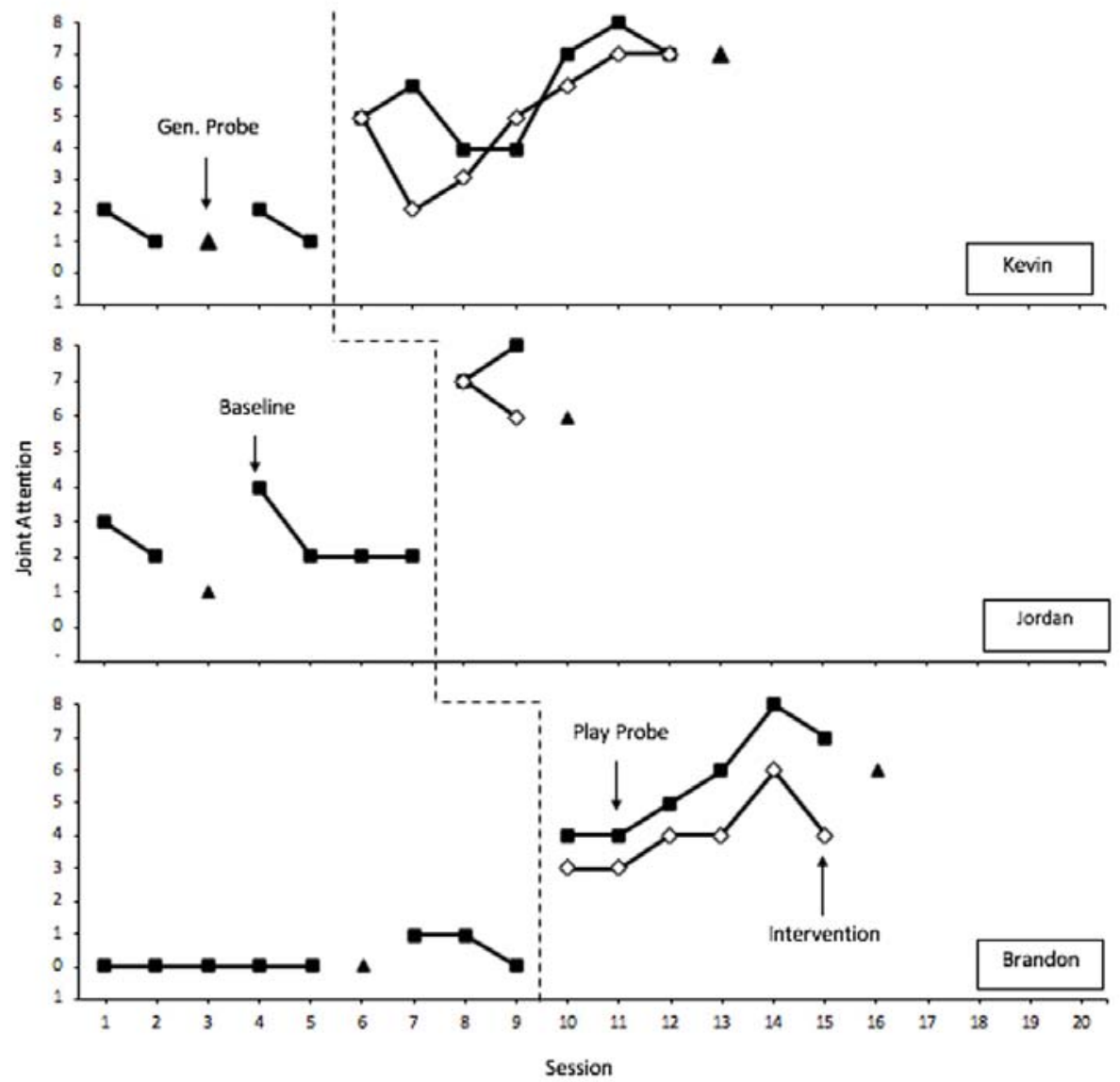

Note. X-axis represents the number of sessions. Y-axis represents the number of trials per session. Closed squares represent the child's joint attention during baseline and during trial-based play probes following intervention sessions. Closed triangles represent the child's joint attention during generalization probes. Open diamonds represent the child's joint attention during the functional toy play intervention sessions. 


\section{iejee $\sqrt{2}$}

play intervention. Jordan met criterion after two play intervention sessions. During intervention, Jordan's joint attention significantly increased in the functional play intervention and play probes from baseline levels. Treatment gains were generalizable to an unfamiliar setting with an unfamiliar person and untrained stimuli.

\section{Brandon}

In baseline, Brandon demonstrated very few joint attention engagements (see panel 3, Figure 1). Following the onset of the play intervention, Brandon exhibited steady gains in joint attention and met criterion (e.g., 7 out of 8 opportunities) within 6 functional play intervention sessions. During intervention, Brandon's joint attention increased in post play probes from baseline levels. For Brandon, joint attention consistently occurred at higher rates in the play probe sessions than the functional toy play intervention sessions. Brandon's increase in joint attention was also exhibited in generalization probes conducted in an unfamiliar setting with an unfamiliar adult and untrained stimuli.

\section{Functional Play Behaviors}

Kevin

During baseline, Kevin exhibited very few functional play behaviors (see panel 1, Figure 2). With the implementation of the functional play intervention, however, Kevin's functional toy play across the 8 opportunities steadily increased. Kevin's play behaviors remained high through the intervention phase of the study. In the play probes, Kevin's play behaviors reached criterion levels (e.g., 7 out of 8 opportunities). Treatment gains generalized to an unfamiliar setting with an unfamiliar person and untrained stimuli.

\section{Jordan}

Jordan's functional play behaviors (see panel 2, Figure 2) remained consistently low during baseline, but immediately increased upon the introduction of the play intervention. Additionally, Jordan's play behaviors increased and reached criterion levels in the play probes. Treatment gains were generalizable to an unfamiliar setting with an unfamiliar person and untrained stimuli.

\section{Figure 2}

Functional Toy Play

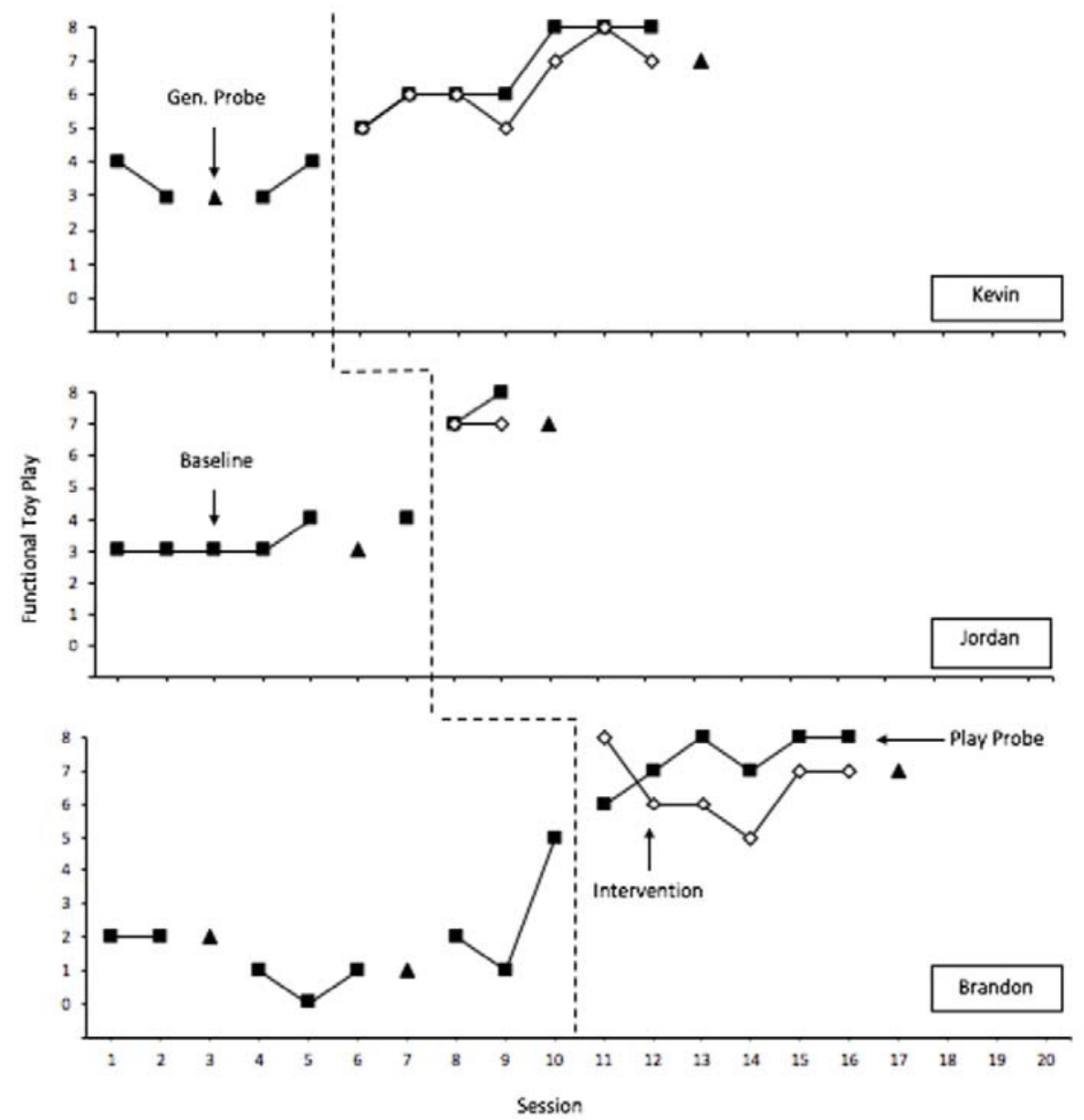

Note. X-axis represents the number of sessions. Y-axis represents the number of trials per session. Closed triangles represent the child's functional toy play behavior during baseline and during trial-based play probes following intervention sessions. Closed squares represent the child's functional toy play behavior during generalization probes. Open circles represent the child's functional toy play behavior during the functional toy play intervention sessions. 


\section{Brandon}

In baseline, Brandon demonstrated very few functional play behaviors (see panel 3, Figure 2). Following the onset of the functional play intervention, Brandon exhibited immediate gains in play behaviors, which remained consistent across his 6 play intervention sessions. Brandon's play behaviors increased from baseline levels in the play probes. For Brandon, functional toy play consistently occurred at higher rates in the play probe sessions than the functional toy play intervention sessions Brandon's increase in play behavior was also exhibited in post-intervention generalization probes conducted in an unfamiliar setting with an unfamiliar person and untrained stimuli.

\section{Discussion}

The present study supports the efficacy of a functional play intervention for increasing joint attention and, expectedly, functional toy play for children with ASD. Following the implementation of the functional play intervention, all three children with ASD showed increases in joint attention and functional play behaviors and met learning criterion by demonstrating 7 correct joint attention engagements out of 8 opportunities across two consecutive play probes that followed the functional play intervention sessions. Of the three children, one child met learning criterion by demonstrating gains in joint attention after only two play intervention sessions; the other two children met learning criterion within 6-7 intervention sessions. All three children demonstrated generalization of increased joint attention and functional play behavior with an unfamiliar person in an unfamiliar setting and novel stimuli, following the completion of treatment.

The present study diverges from the current joint attention literature in that it taught joint attention via a functional play intervention. While White and colleagues (2011) discussed that using play as a context is "best practice" for teaching joint attention skills, research had yet to assess the efficacy of teaching play as a means to acquire joint attention. Previous literature does support the use of play as the context for teaching joint attention, and there is evidence of a link between joint attention and play skills for children with ASD (Miendl \& Cannella-Malone, 2011; White et al., 2011). Play is a highly motivating behavior for both children with and without ASD (Charlop, Lang \& Rispoli, 2018), and therefore using play as the vehicle to teach joint attention skills was a natural conclusion (Boutot et al., 2005; Kasari et al., 2010; Whalen \& Schreibman, 2003). Play provides a good means for teaching joint attention as it allows for the programming of common stimuli (i.e., familiar toys) and shared control with turn taking (White et al., 2011), includes a play partner (White et al., 2011), and provides natural social consequences. Play often requires a play partner (i.e., parent, sibling, a therapist) to model appropriate play (e.g., turn-taking, functional toy play) for the child, thus providing natural opportunities for the child to engage in joint attention with their social-communicative partner and the object of interest (i.e., the toy). As a result, play benefits generalization.

In the present study, as functional play was taught, it likely promoted the occurrence of joint attention in that joint attention was a natural part of learning functional play. During functional play, attending to both the play partner and the toy (i.e., the object of interest) is a necessary part of the functional play intervention in that it requires the child to imitate modeled play. Furthermore, the post intervention play probes demonstrated that joint attention increased as functional toy play increased after the direct functional play intervention.

Moreover, the results demonstrated generalization of both behaviors to an unfamiliar setting with an unfamiliar person and novel stimuli. Although the measurement of generalization was minimal in the present study and consisted of only one probe (generalization and not maintenance), treatment gains were generalizable across all three children. Previous literature had not seen generalizable results (e.g. Kasari et al., 2010). Thus, it appears that play may benefit the generalization of joint attention skills as it includes naturally maintaining consequences (White et al., 2011). In much of the previous literature on joint attention, the use of edible and tangible reinforcers were often used (Miendl \& Cannella-Malone, 2011; White et al., 2011). However, the present study embedded the reinforcement of joint attention within a functional play intervention. While joint attention requires sharing social attention with a socialcommunicative partner, play requires access to toys (Warreyn et al., 2014; White, et al, 2011). Thus, within the functional play paradigm, access to the toy(s) and shared social attention with a social-communicative play partner are naturally maintaining reinforcers. As a result, learning joint attention within play may have facilitated generalizations.

The present study extends joint attention research by providing evidence that older school-aged, minimallyverbal children with ASD benefit from joint attention and play interventions. Research has long focused on teaching joint attention to toddlers and preschoolaged children with ASD (Kasari et al., 2006; Miendl \& Cannella-Malone, 2011; Murza et al., 2016; White et al., 2011). To date there is no research that has included targets of joint attention for older children with minimal verbal skills as in the present study. Understandably, past research has focused on younger children because of the strong link between joint attention and early development of more complex language, play and social behaviors (Baron-Cohen, 1991; Charman et 
al., 2000; Charman et al., 2003; Charman et al., 1997; Delinicolas \& Young, 2007; Mundy et al., 2007; Murray et al., 2008; Rollins \& Snow, 1998; Schertz \& Odom, 2004; Tomasello \& Farrar, 1986). However, older children might also benefit from joint attention interventions; more specifically, with increased joint attention skills, these children may see increases in more complex social and language behaviors. These results highlight the continued benefits of targeting joint attention, an important yet complex skill, in older children with ASD. In summary, the present study also demonstrated that joint attention can successfully be taught via a functional play intervention to children who were considered minimally verbal or "non-verbal" such that they did not exhibit speech at the time of the study and did not communicate reliably via an alternative/augmentative communication device. The children demonstrated interfering behaviors such as stereotypy, inattentiveness, aggression, tantrums, and other problem behaviors. Accordingly, they would be considered a challenging group of participants in need of intervention for both functional play and joint attention.

The present study also included participants from three divere ethnicities. It is rare in the ASD literature that children from a variety of communities are identified. Such cross cultural identification. and inclusion is important as we move forward as a field in the 21 st century.

The results of the present study also suggest that it is "not too late" to target joint attention in older, schoolaged children. The results of this study raise some suggestions for future research. First, it is necessary to replicate these findings, and perhaps determine if older persons (i.e., adolescents, adults) with ASD can increase and acquire joint attention behavior. This is especially important given the heavily cited relationship between joint attention, play, and language (e.g., Kasari et al., 2006; Miendl \& CannellaMalone, 2011; Murza et al., 2016; White et al., 2011). While there was no direct data available from the present study, it was anecdotally noted that all three participants did improve their language skills after participation in the study. Second, research could further investigate the generalization and maintenance of these skills and behaviors more extensively. Third, there is a need for research using natural play partners, including parents, siblings and peers. This might benefit generalization and may provide more opportunities for the child with ASD to practice these skills throughout the day, and not just during intervention sessions (Spector \& Charlop, 2017). In conclusion, the present study provides promising results and empirical support of a functional play intervention to increase joint attention for schoolaged, non-verbal children with ASD.

\section{References}

American Psychiatric Association (2013). Diagnostic and statistical manual of mental disorders (5th ed.). Washington, DC: Author.

Bakeman, R., \& Adamson, L. B. (1984). Coordinating attention to people and objects in motherinfant and peer-infant interaction. Child Development, 55, 1278- 1289.

Baron-Cohen (1991). Precursors to a theory of mind: Understanding attention in others. In A. Whiten (Ed.), Natural theories of mind: Evolution, development and simulation of everyday mindreading (p. 233-251). Basil Blackwell.

Bates, E., Benigni, L., Bretherton, I., Camaioni, L. \& Volterra, V. (1979). The emergence of symbols: Cognition and communication in infancy. New York: Academic Press.

Boutot, E., Guenther, T., \& Crozier, S. (2005). Let's Play: Teaching play skills to young children with autism. Education and Training In Developmental Disabilities, 40(3), 285-292.

Charlop, M.H, Lang, R. \& Rispoli, M. (2018). Evidencebased approaches to teaching play and social skills to children with autism spectrum disorder. springer.

Carpenter, M., Pennington, B. F., \& Rogers, S. J. (2002). Interrelations among social-cognitive skills in young children with autism. Journal of Autism and Developmental Disorders, 32, 91-106.

Charman, T., Baron-Cohen, S., Swettenham, J., Baird, G., Drew, A., \& Cox, A. (2000). Testing joint attention, imtation, and play as infancy precursors to langauge and theory of mind. Cognitive Development, 15(4), 481-498.

Charman, T., Baron-Cohen, S., Swettenham, J., Baird, G., Drew, A., \& Cox, A. (2003). Predicting language outcome in infants with autism and pervasive developmental disorder. International Journal of Language and Communication Disorders, $38,265-285$.

Charman, T., Swettenham, J., Baron-Cohen, S., Cox, A., Slonims, V., Wheelwright, S., et al. (1997). Infants with autism: An investigation of empathy, pretend play, joint attention, and imitation. Developmental Psychology, 33, 781-789. 
Kasari, C., Freeman, S., \& Paparella, T. (2006). Joint attention and symbolic play in young children with autism: A randomized controlled intervention study. Journal of Child Psychology and Psychiatry, 47(6), 611-620.

Kasari, C., Freeman, S., Paparella, T., \& Jahromi, L.B. (2008). Language outcome in autism: Randomized comparison of joint attention and play interventions. Journal of Child Psychology and Psychiatry, 76(1), 125-137.

Kasari, C., Gulsrud, A.C., Wong, C., Kwon, S. \& Locke, J. (2010). Randomized controlled caregiver mediated joint attention engagement intervention for toddlers with autism. Journal of Autism and Developmental Disorders, 40, 10451056.

MacDonald, R., Anderson, J., Dube, W.V., Geckeler, A., Green, G., Holcomb, W., et al. (2006). Behavioral assessment of joint attention: A methodological report. Research in Developmental Disabilities, 27, 138-150.

Miendl, J.N. \& Cannella-Malone, H.I. (2011). Initiating and responding to joint attention bids in children with autism: A review of the literature. Research in Developmental Disabilities 32, 1441-1454.

Mundy, P., Sigman, M. \& Kasari, C. (1994). Join attention, developmental level and symptom presentation in autism. Development and Psychopathologym, 6, 389-401.

Mundy, P. (1995). Joint attention and social-emotional approach behavior in children with autism. Development and Psychopathology, 7, 63-82.

Mundy, P., Sigman, M., Ungerer, J. \& Sherman, T. (1986). Defining the social deficits of autism: The contribution of nonverbal communication measures. Journal of Child Psychology and Psychiatry, 27, 657-669.

Nock, M. K. (2002). A multiple-baseline evaluation of the treatment of food phobia in a young boy. Journal of Behavior Therapy And Experimental Psychiatry, 33(3-4), 217-225.

Pierce, K. \& Schreibman, L. (1995). Increasing complex social behaviors in children with autism: Effects of peer-implemented pivotal response training. Journal of Applied Behavior Analysis, 23, 285295.
Osterling, J., \& Dawson, G. (1994). Early recognition of children with autism: A study of first birthday home videotapes. Journal of Autism and Developmental Disorders, 24, 247-256.

Spector, V. \& Charlop, M. H. (2017). A Sibling-Mediated Intervention for Children with Autism Spectrum Disorder: Using the Natural Language Paradigm (NLP). Journal of Autism and Developmental Disorders, 1-15.

Taylor, B. A., \& Hoch, H. (2008). Teaching children with autism to respond to and initiate bids for joint attention. Journal Of Applied Behavior Analysis, 41(3), 377-391. doi:10.1901/jaba.2008.41-377

Tomasello, M. \& Farrar, M. (1986). Joint attention and early language. Child Development, 57, 14541463.

Warryen, P., Van Der Paelt, S., \& Roeyers, H. (2014). Socialcommunicative abilities as treatment goals for preschool children with autism spectrum disorder: The importance of imitation, joint attention, and play. Developmental Medicine and Child Neurology, 56, 712-716.

Whalen, C. \& Schreibman, L. (2003). Joint attention training for children with autism using behavior modification procedures. Journal of Child Psychology and Psychiatry, 44, 456-468.

White, P.J., O'Reilly, M., Streusand, W., Levine, A., Sigafoos, J., Lancioni, G., Fragale, C., Pierce, N. \& Aguilar, J. (2011). Best practices for teaching joint attention: A systematic review of the intervention literature. Research in Autism Spectrum Disorders, 5(4), 1283-1295. 